\title{
Gait Analysis Platform for Measuring Surgery Recovery
}

\author{
Marc CODINA ${ }^{\mathrm{a}, 1}$, Manuel NAVARRETE ${ }^{\mathrm{a}}$, Ashkan REZAEE ${ }^{\mathrm{a}}$, David CASTELLS- \\ RUFAS $^{\mathrm{a}}$, Maria Jesús TORRELLES ${ }^{\mathrm{a}}$, Stefan BURKARD ${ }^{\mathrm{b}}$, Holger ARNDT ${ }^{\mathrm{b}}$, Sabine \\ DREVET $^{\mathrm{c}}$, Medhi BOUDISSA ${ }^{\mathrm{c}}$, Jerome TONETTI ${ }^{\mathrm{c}}$, Isabelle MARQUE ${ }^{\mathrm{d}}$, Alexandre \\ MOREAU-GAUDRY ${ }^{\mathrm{d}}$, Armand CASTILLEJO ${ }^{\mathrm{e}}$, Jordi CARRABINA ${ }^{\mathrm{a}}$ \\ ${ }^{a}$ CEPHIS. MiSE Dpt. Universitat Autònoma de Barcelona, Bellaterra, Spain \\ ${ }^{\mathrm{b}}$ SpringTechnoGMBh, Bremen, Germany \\ ${ }^{c}$ Dpt. of Orthopedic and Trauma Surgery. CHU Grenoble-Alpes, La Tronche, France \\ 'INSERM. CHU Grenoble-Alpes, CIC1404, Grenoble, France \\ ${ }^{e}$ STMicrolectronics, Grenoble, France
}

\begin{abstract}
Gait analysis has evolved significantly during last years due to the great development of the Medical Internet of Things (MIoT) platforms that allow an easy integration of sensors (inertial, magnetic and pressure in our case) to the complex analytics required to compute, not only relevant parameters, but also meaningful indexes. In this paper, we extend a previous development based on a fully wireless pair of insoles by implementing an updated version with more reliable and userfriendly devices, smartphone app and web front-end and back-end. We also extend previous work focused on fall analysis (with the corresponding fall risk index or FRI) with the proposal of a new surgery recovery index (SRI) to account for the individual speed recovery speed that can be measured either at clinical facilities or at home in a telemedicine environment or while doing daily life activities. This new index can be personalized for different types of surgeries that affect gait such as hip, knee, etc. This paper presents the case of hip recovery and is built on top of the clinical standard SPPB test and allows obtaining quantitative parameters directly from the sensors.
\end{abstract}

Keywords. Gait Analysis, Insoles, Hip surgery recovery, Recovery Index, Medical Internet-of-Things

\section{Introduction}

Wearables have been significantly relevant in increasing the motivation for wellbeing all around the world. It should be agreed that this should improve the general health care situation for all age ranges and profiles. Wearable recordings started with counting steps and progressively added new measures (oxygen saturation, pulse, etc.) to even more complex functionalities (electrocardiographic analysis, fall detection, gait analysis (e. g. for runners). Wearables have the advantage that can take and store continuous measurements on health parameters. These parameters are not yet being intensively used and/or integrated in the health records of the users or patients nor in the health platforms

1 Corresponding Author: Marc Codina; Universitat Autònoma de Barcelona, Centre de Prototips i Solucions Hardware-Software, CEPHIS. Escola d'Enginyeria, Campus UAB, 08193 Bellaterra, Spain; Email: Marc.Codina@uab.cat. 
from public and private health providers what can be considered as a loss of useful information. It is commonly agreed that some of the reasons are the different precision of the different sensors used or the complexity of the integration of patients' data into the health records. Nonetheless, the evolution of the sensor technology and related calibration procedure plus the increasing access to platform providers through APIs easier those walls. Still, it remains one relevant issue: how to move or transform existing criteria and protocols based on measurements done in clinical installations to integrate this existing data in the health records of the patients. This platform will be used for validating relevant data (raw or derived) according to the golden standards. In this paper, we present a proposal for such framework devoted to measure the degree of recovery after a surgery that affects gait.

Gait analysis has become a widely used clinical tool, enabling clinicians to provide better patient care. The analysis of the gait of patients offers new opportunities for diagnosis and treatment of walking problems and to find patterns in gait $(1,2)$.

Our hypothesis if that with semi-automated gait analysis it is possible to evaluate the recovery status of a patient from surgery and provide a new Surgery Recovery Index (SRI) to quantify the individual rate of recovery. This analysis can be carried out in both ways, in the clinical facility or at home while performing activities of daily life. In the former WIISEL project, we worked on the analysis of falls and the elaboration of a Fall Risk Index (FRI), since falls are a major cause of injuries for the elderly people (3). This project was also validated by several clinical studies (4). Nonetheless, the insole prototype was far from a final product concerning reliability of its components and integration.

In the scope of the SERENE project (5), we present an updated MIoT platform to collect real-time gait data from users wearing a custom pair of wireless insoles, oriented to the analysis of the recovery process for patients after a surgery that affects their gait that is currently measured by the score of a given test and we propose to measure by defining a numerical surgical recovery index.

\section{The SERENE Platform}

The SERENE project is a collaborative project with a consortium of 20 partners from France, Germany and Spain with the goal of providing better remote care and diagnostic tools using Medical IoT technologies. Our contribution is centered on providing a platform for gait analysis composed of a pair of smart-insoles and a smartphone per patient, a cloud data-base, a software platform for the analytics and a web access for 3 types of users (patients, caregivers / relatives and clinicians). 

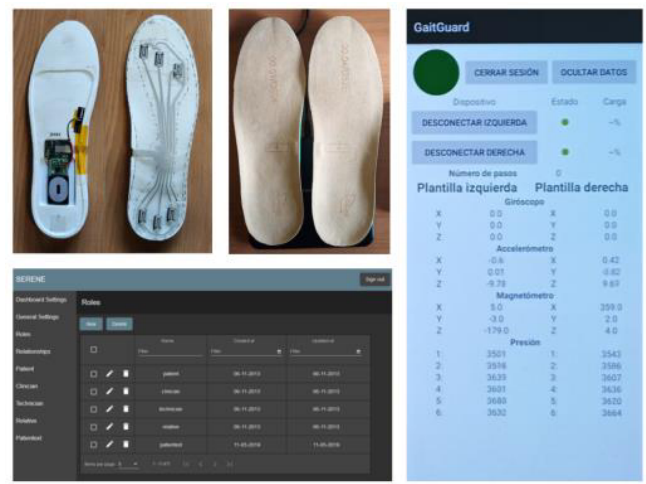

Figure 1. Platform components: Insoles, Android APP and Web interface

The wearable insoles were designed to look and feel like regular insoles in terms of comfort with the support of a commercial insole maker: Flexor. The internal electronics were separated into two flexible parts for power and computation/communication plus two antennas, allowing them to be adjusted to the desired shape or to flex during use. The communication with the mobile phone is via Bluetooth Low Energy (BLE). The insoles have no charging connectors, as they are equipped with Qi wireless charging. An Inertial Measurement Unit (IMU) plus a pressure sensor layer are used as the source of data. The IMU outputs acceleration and gyroscope data, each 3-axis, together with selfcalibrated orientation in real time. The pressure sensor layer includes 6 force-sensitive resistors (FSRs) positioned under the heel and the metatarsal. Sensor data acquisition system is 33 samples per second for all sensors while the IMU has 14 bits to16 bits resolution while pressure sensors have 10 bits. The IMU includes a Cortex-M0 processor able to compute quaternions that will also be used for gait analysis. Captured data is formatted in 20 bytes packets and sent to the smartphone through Bluetooth Low Energy (BT5.1)

An application in the smartphone is used to monitor and display the data acquired from the pressure sensors and the information generated by the IMU. The bidirectional communication between the smartphone and the smart insoles is based on Bluetooth Low Energy using a custom GATT profile. The data collected from both smart insoles is processed and appended into a data file that is stored for later analysis.

In addition, the mobile phone acts as a bridge between the insoles connected via Bluetooth and the cloud server which we access via LTE/5G or Wi-Fi. This communication with the server allows access to the patient's personal profile, to retrieve statistical data of the user as well as to send the files with the sensor data captured from the insoles.

The data analysis platform consists of a back-end in which a service responds to requests from the mobile application once the clinical trial recording session is finished. It stores collected data in a database from which 49 parameters related to the gait analysis are computed.

The front-end allows the access of the different users to the processed data through a dashboard designed according to specific profiles: clinician, patient and relative. The user interface graphically displays the gait analysis data and allows the selection of relevant parameters by the clinician what allows personalizing the medical treatment according to the status of the patient. 


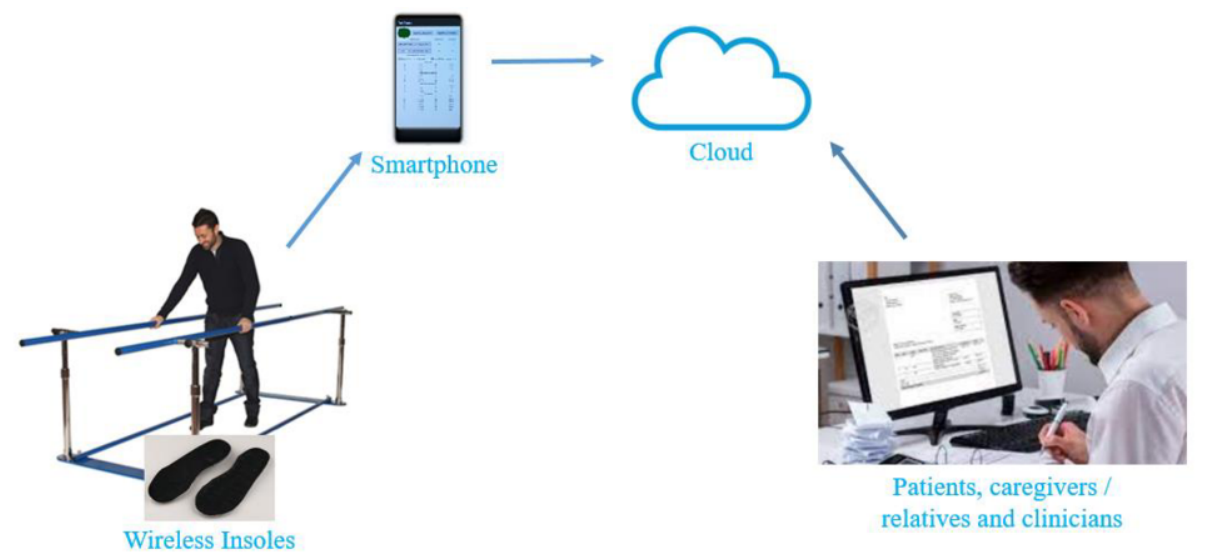

Figure 2. Diagram of the SERENE platform

\section{Gait analysis.}

Using the new sensors implemented in this revision of the technological part, the number of gait features extracted after processing the sensor data have been extended from 26 to 49. The gait analysis framework has been designed and organized into layers, each one having a specific functionality. The framework consists of five main layers: Raw data processing; Gait parameter definition; Pattern recognition and feature extraction; Gait parameter processing and Surgery Recovery Index (SRI) and exploitation of gait analysis results.

From IMU sensors data, we estimate the position and orientation of the foot with respect to the world. Madgwick's gait tracking algorithms were implemented to determine the position of the patient as they move either through a controlled environment as a clinical facility (6) or at home. Among the parameters that are extracted with the data obtained from the IMU (3D accelerometer, 3D gyroscope and 3D magnetometer) and pressure sensors, we can find the following ones: Cadence, Double Support, Single Support, Swing Phase, Stance Phase, Gait Symmetry, Heel Strike Force Slope, Average Acceleration Amplitude Mediolateral. Some similar approaches can be found in the literature (7).

\section{Surgery Recovery}

Both surgery recovery and fall risk assessment are based on the analysis of gait parameters. The main differences among them are: the temporal scale of the patients' evolution (usually faster and more deterministic in recovery processes and slower and unpredictable in the evolution of the elderly) and also the relevant gait parameters and their combination to obtain the index that is much more related to the specific surgery rather than to the individual health state as in the case of the elderly).

We focused this new version of the platform in hip surgery recovery. According to the literature, several aspects related to gait can be used to evaluate recovery improvement (8): walking velocity, stride length, range of hip motion and hip abduction 
moments. Other authors consider further measurements that can be considered such as: Muscle strength (voluntary isometric force of the hip and knee muscles related to walking ability) (9-11) or balance (postural stability) (12). Some of these parameters must be directly obtained or estimated by the observation of the clinician while others can be provided by our gait analysis platform. Our idea of SRI is to indicate whether the patient is progressing faster, equal or slower than the average for a given recovery time after surgery.

After evaluating the different tests proposed in the literature (13-15), we decided to use SPPB (16) as the basis for our tests. This test evaluates balance, gait speed and the ability to get up and down a chair (as indicator of strength) by measuring the time of each activity. It currently provides a combination of scores, giving more precision in the differences in stride and balance (17). The values obtained from IMU and pressure sensors for each one of the three stages of the SPPB test will be captured with higher confidence. In the case of balance, we use a posturometer as a gold standard to fine tune our algorithms.

We consider two different scenarios. The first one is related to rehabilitation tracking in clinical facilities, where multiple patients will be able to use the insoles (according to their feet size) in their rehabilitation routines and clinicians can analyze the data obtained through our platform. This leads to a sporadic use of the system. The second model focuses on domestic use, where the patient wears a pair of insoles on their daily life activities. Then, clinicians track that patient at any time on their dashboards. When any anomaly is detected, the clinician will contact the patient for a more detailed follow-up. This model can also be used for remote monitoring of specific exercises in telemedicine scenarios that are becoming popular during the COVID-19 pandemic.

The hip SRI complements the existing SPPB test with the parameters obtained by the insoles (pressure and motion) for each stage of the test. Studies about the weight of the parameters in the index and the way to personalize them according to the patient will be carried out at later research.

\section{Conclusion}

This paper presents a solution of a platform for surgical recovery analysis composed of a pair of fully wireless insoles, a smartphone app and a cloud solution that includes both database and web personalized dashboards. We proposed a new Surgical Recovery Index (SRI) to account for the evolution of the patient after a surgery that affects its gait.

We apply this procedure to hip surgery recovery upon the existing SPPB test, whose score will be complemented by the data obtained and computed from sensor parameters, thus becoming easy to interpret in terms of recovery rate, that depends on the health state and specific surgery done to every person.

This SRI model can be extended to other surgeries that affect gait such as hip, knee, achilles, etc. $(8,18,19)$. In some cases, when data is available, that index will consider the status before the surgery or even before the lesion (e.g. in sports).

This platform can be deployed for remote surgery recovery in several scenarios, all of them under the premise that secure servers must hold medical records. We initially plan to use the devices in both clinical facilities and at patients' home. In this second case, two models are available: a telemedicine scenario to proceed with tests supervised remotely by clinicians and an unsupervised scenario based on daily living activities with the automatic identification of relevant situations to compute SRI. It is planned to start 
soon the clinical trials to analyze the recovery status of several patients at Grenoble hospital.

\section{References}

[1] Pagano G, D’Addio G, De Campi M, Donisi L, Biancardi A, Cesarelli M. Rehabilitation Outcome in Patients undergone Hip or Knee Replacement Surgery using Inertial Technology for Gait Analysis. IEEE Med Meas Appl MeMeA 2020 - Conf Proc. 2020;

[2] Ghent F, Mobbs RJ, Mobbs RR, Sy L, Betteridge C, Choy WJ. Assessment and Post-Intervention Recovery After Surgery for Lumbar Disk Herniation Based on Objective Gait Metrics from Wearable Devices Using the Gait Posture Index. World Neurosurg [Internet]. 2020;142:e111-6. Available from: https://doi.org/10.1016/j.wneu.2020.06.104

[3] Arndt H, Burkard S, Talavera G, Garcia J, Castells D, Codina M, et al. Real-time constant monitoring of fall risk index by means of fully-wireless insoles. Stud Health Technol Inform. 2017;237:193-7.

[4] Bravo J, Cook D, Riva G. Ambient intelligence for health environments. Vol. 64, Journal of Biomedical Informatics. 2016. 15-25 p.

[5] SERENE-IoT site [Internet]. Available from: https://penta-eureka.eu/project-overview/penta-call$1 /$ serene-iot/

[6] Madgwick SOH, Harrison AJL, Vaidyanathan R. Estimation of IMU and MARG orientation using a gradient descent algorithm. IEEE Int Conf Rehabil Robot. 2011;

[7] Nouredanesh M, Godfrey A, Howcroft J, Lemaire ED, Tung J. Fall risk assessment in the wild: A critical examination of wearable sensor use in free-living conditions. Gait Posture [Internet]. 2021;85(December 2019):178-90. Available from: https://doi.org/10.1016/j.gaitpost.2020.04.010

[8] Ewen AM, Stewart S, St Clair Gibson A, Kashyap SN, Caplan N. Post-operative gait analysis in total hip replacement patients-A review of current literature and meta-analysis. Gait Posture [Internet]. 2012;36(1):1-6. Available from: http://dx.doi.org/10.1016/j.gaitpost.2011.12.024

[9] Rantanen T, Era P, Heikkinen E. Maximal Isometric Strength and Mobility among 75-year-old Men and Women. Age Ageing [Internet]. 1994 Mar 1;23(2):132-7. Available from: https://doi.org/10.1093/ageing/23.2.132

[10] Rantanen T, Avlund K, Suominen H, Schroll M, Frändin K, Pertti E. Muscle strength as a predictor of onset of ADL dependence in people aged 75 years. Aging Clin Exp Res. 2002;14(3 Suppl):10-5.

[11] Manini TM, Clark BC, Nalls MA, Goodpaster BH, Ploutz-Snyder LL, Harris TB. Reduced physical activity increases intermuscular adipose tissue in healthy young adults. Am J Clin Nutr. 2007;85(2):37784.

[12] Fiatarone MA, Marks EC, Ryan ND, Meredith CN, Lipsitz LA, Evans WJ. High-Intensity Strength Training in Nonagenarians: Effects on Skeletal Muscle. JAMA J Am Med Assoc. 1990;263(22):302934.

[13] Soares Menezes KVR, Auger C, de Souza Menezes WR, Guerra RO. Instruments to evaluate mobility capacity of older adults during hospitalization: A systematic review. Arch Gerontol Geriatr [Internet]. 2017;72(October 2016):67-79. Available from: https://doi.org/10.1016/j.archger.2017.05.009

[14] Shumway-Cook A, Brauer S, Woollacott M. Predicting the probability for falls in community-dwelling older adults using the timed up and go test. Phys Ther. 2000;80(9):896-903.

[15] Tinetti M. Performance-oriented assessment of mobility problems in elderly patients. J Am Geriatr Soc. 1986;34(2):119-26.

[16] Guralnik JM, Simonsick EM, Ferrucci L, Glynn RJ, Berkman LF, Blazer DG, et al. A short physical performance battery assessing lower extremity function: Association with self-reported disability and prediction of mortality and nursing home admission. Journals Gerontol. 1994;49(2).

[17] Lopes KJY, Pin ADS, Miranda RM da S, Ghelfi A. Comparison of the Tinetti and Short Physical Performance Battery test specificities for detecting neuromotor pathologies. ConScientiae Saúde. 2019;18(1):1-7.

[18] McClelland JA, Webster KE, Feller JA. Gait analysis of patients following total knee replacement: A systematic review. Knee. 2007;14(4):253-63.

[19] Don R, Ranavolo A, Cacchio A, Serrao M, Costabile F, Iachelli M, et al. Relationship between recovery of calf-muscle biomechanical properties and gait pattern following surgery for achilles tendon rupture. Clin Biomech. 2007;22(2):211-20. 